\title{
Erratum to: The Transformation of Georgia from 2004 to 2012
}

\author{
Dimitri Gvindadze
}

\section{Erratum to:}

D. Gvindadze, The Transformation of Georgia from 2004 to 2012, https://doi.org/10.1007/978-3-319-59201-5

The original version of the book was inadvertently published with 6 endorsements instead of 8 in Frontmatter. The erratum book has been updated with the change. 